\title{
Quasiparticle photoemission intensity in doped two-dimensional quantum antiferromagnets
}

\author{
F. Lema and A. A. Aligia \\ Centro Atómico Bariloche and Instituto Balseiro, \\ Comisión Nacional de Energía Atómica, \\ 8400 Bariloche, Argentina
}

\begin{abstract}
Using the self-consistent Born approximation, and the corresponding wave function of the magnetic polaron, we calculate the quasiparticle weight corresponding to destruction of a real electron (in contrast to creation of a spinless holon), as a funtion of wave vector for one hole in a generalized $t-J$ model and the strong coupling limit of a generalized Hubbard model. The results are in excellent agreement with those obtained by exact diagonalization of a sufficiently large cluster. Only the Hubbard weigth compares very well with photoemission measurements in $\mathrm{Sr}_{2} \mathrm{CuO}_{2} \mathrm{Cl}_{2}$.

PACS numbers: 75.10.Jm, 79.60.-i, 74.72.-h
\end{abstract}


The problem of a single hole in an antiferromagnetic background has been a subject of considerable interest since the discovery of high- $T_{c}$ systems . One of the most powerful tools for this study is the self-consistent Born approximation (SCBA) [1] [] has been obtained between the position of the lowest pole of the holon Green function of the SCBA and the quasiparticle dispersion obtained by exact diagonalization of small systems [3 5]. An important advance in the understanding of the SCBA has been the explicit construction of the corresponding wave function by Reiter [6].

The interest on the problem has been revived by recent angle-resolved photoemission experiments on insulating $\mathrm{Sr}_{2} \mathrm{CuO}_{2} \mathrm{Cl}_{2}$, in which the hole dispersion and quasiparticle weight have been measured [7]. While it was clear that the "bare" $t-J$ model was unable to explain the observed dispersion, several works have appeared fitting the experimental dispersion using generalized $t-J$ models [5,8,9, a generalized Hubbard model [10] and the spinfermion (or Kondo-Heisenberg) model for the cuprates [11. Except for the fact that the band width is $\sim 10 \%$ narrower than the experimental result if the experimental value of $J$ is taken [12], the generalized $t-J$ model including hopping to second and third NN and the three-site term $t^{\prime \prime}$, reproduces well the experimental dispersion [5.9] and also other properties of the spin-fermion and three-band Hubbard models [12]. A consistent picture of the observed spin and charge excitations has been obtained using a generalized one-band Hubbard model [10].

However, very little attention has been devoted to the explanation of the intensity of the observed quasiparticle peaks. This task is difficult for the following reasons: i) exact results for quasiparticle intensities in sufficiently large clusters (containing more than 16 unit cells, as discussed below) exist only for the "bare " $t-J$ model and only at a few wave vectors. ii) The SCBA provides the Green function of the spinless holon, while the Green function of the real particles contain spin-wave excitations and simple decoupling approximations do not provide reasonable results. The holon weights are the same for wave vectors differing in $(\pi, \pi)$ contrary to experiment. iii) While a lot of work has been devoted to the mapping of the three-band Hubbard model for the cuprates to low-energy effective models, less attention has 
been devoted to the mapping of the corresponding operators [12,14, 15]. This information as well as the photoionization cross sections for $\mathrm{Cu}$ and $\mathrm{O}$ are necessary if accurate weights are wished.

In this paper we calculate the photoemission quasiparticle weight for removing an electron, as a function of wave vector in generalized $t-J$ and strong-coupling Hubbard models, using the SCBA and the wave function of the polaron [6]. The Hamiltonian has the form

$$
\begin{aligned}
H= & -\sum_{i \delta \sigma} t_{\delta} c_{i+\delta \sigma}^{\dagger} c_{i \sigma}-t^{\prime \prime} \sum_{i \eta \neq \eta^{\prime} \sigma} c_{i+\eta^{\prime} \sigma}^{\dagger} c_{i \eta \sigma}\left(\frac{1}{2}-2 \mathbf{S}_{i} \cdot \mathbf{S}_{i+\eta}\right) \\
& +\frac{J}{2} \sum_{i \eta \sigma}\left(\mathbf{S}_{i} \cdot \mathbf{S}_{i+\eta}-\frac{1}{4} n_{i} n_{i+\eta}\right) .
\end{aligned}
$$

The first term contains hopping to first, second and third nearest neighbors (NN) with parameters $t_{1}, t_{2}, t_{3}$ respectively. The first $\mathrm{NN}$ of site $i$ are labeled as $i+\eta$. Eq. (11) is obtained from a standard canonical transformation of a Hubbard model with hoppings $t_{1}, t_{2}, t_{3}$, if (complicated) terms smaller than $t^{\prime \prime}=t^{2} / U$ are neglected [16]. The difference between generalized $t-J$ and strong-coupling Hubbard models is the meaning of the operator $c_{i \sigma}$, as explained below. The Hamiltonian can be written in terms of spinless fermions and spin-wave operators $[1,4,16]$. We adopt the procedure and notation used by Martínez and Horsch [3], slightly generalized to include second and third NN hoppings and the threesite term [16]: The sublattice $A$ is defined as that of positive magnetization. The spins of sublattice $\mathrm{B}$ are rotated $180^{\circ}$ around the $\mathrm{x}$ axis. In this way the Neel state is converted into a fully polarized ferromagnetic state, restoring the translational symmetry of the nonmagnetic state at the price of losing the conservation of spin. Then, the $c_{i \uparrow}$ operator is defined as a spinless holon creation operator $h_{i}^{\dagger}$, while $c_{i \downarrow}$ becomes a composite operator involving a local spin deviation $a_{i}$. The result of both operations is the following representation:

$$
\begin{array}{ll}
c_{i \uparrow}=h_{i}^{\dagger}, \quad c_{i \downarrow}=h_{i}^{\dagger} a_{i}, & \text { if } i \in \mathrm{A} \\
c_{i \uparrow}=h_{i}^{\dagger} a_{i}, \quad c_{i \downarrow}=h_{i}^{\dagger}, & \text { if } i \in \mathrm{B} .
\end{array}
$$

In the exchange part (last term of Eq. (1)) the fermion occupation numbers are averaged and the bosonic quadratic part is diagonalized by a standard canonical transformation: 


$$
\alpha_{q}=u_{q} a_{q}-v_{q} a_{-q}^{\dagger}
$$

where $u_{q}^{2}=v_{q}^{2}+1=1 / 2+1 /\left(2 \nu_{q}\right), \quad \nu_{q}=\left(1-\gamma_{q}^{2}\right)^{1 / 2}, u_{q}>0, \operatorname{sgn}\left(v_{q}\right)=\operatorname{sgn}\left(\gamma_{q}\right)$, and $\gamma_{q}=$ $\left(\cos q_{x}+\cos q_{y}\right) / 2$. Retaining only linear terms in spin deviations for the rest of Eq. (1)), the Hamiltonian becomes:

$$
\begin{aligned}
H= & E_{J}^{0}+\sum_{q} \omega_{q} \alpha_{q}^{\dagger} \alpha_{q}+\sum_{k} \epsilon_{k} h_{k}^{\dagger} h_{k} \\
& +\frac{4 t_{1}}{\sqrt{N}} \sum_{k q} M(k, q)\left(h_{k}^{\dagger} h_{k-q} \alpha_{q}+\text { H.c. }\right)
\end{aligned}
$$

where $E_{J}^{0}$ is a constant, $\omega_{q}=2 J \nu_{q}, \epsilon_{k}=\left(t_{2}+2(1-x) t^{\prime \prime}\right) \epsilon_{2}(k)+\left(t_{3}+(1-x) t^{\prime \prime}\right) \epsilon_{1}(2 k)$ and $M(k, q)=\left(u_{q} \gamma_{k-q}+v_{q} \gamma_{k}\right)$, with $\epsilon_{1}(k)=4 \gamma_{k}$ and $\epsilon_{2}(k)=4 \cos k_{x} \cos k_{y}$. In the present case, the doping $x=0$. The constraint that at the same site there cannot be both a hole and a spin deviation is neglected since it does not affect the results for motion of a hole in a quantum antiferromagnet [3]. The holon Green function $G_{h}(k, \omega)$ is obtained from the self-consistent solution of the following two equations:

$$
\begin{aligned}
\Sigma(k, \omega) & =\frac{4 t_{1}}{N} \sum_{q} M^{2}(k, q) G_{h}\left(k-q, \omega-\omega_{q}\right) \\
G^{-1}(k, \omega) & =\omega-\epsilon_{k}-\Sigma(k, \omega)+i \epsilon .
\end{aligned}
$$

We have solved Eqs. (5) in clusters of $16 \times 16$ and $20 \times 20$ sites. In order to obtain accurate values of the holon quasiparticle weight $Z_{h}$, we have discretized the frequencies in intervals of $\Delta \omega=10^{-4} t_{1}$ and have taken the small imaginary part $\epsilon=5 \Delta \omega$. As an alternative method to that used by Liu and Manousakis [4], we have fitted the part of the spectral weight nearest to the quasiparticle peak by a sum of several Lorentzian functions. The resulting width of the quasiparticle peak was practically identical to $2 \epsilon$ and from its integrated weight we determined $Z_{h}$. We have verified that using this method there are practically no finite-size effects in our clusters.

In the sudden approximation, the angle-resolved photoemission spectrum is proportional to the spectral density of states for $\mathrm{Cu}$ and $\mathrm{O}$ at wave vector $k$. These in turn are related to the imaginary part of the Green function for the generalized $t-J$ operator $c_{k \sigma}$ or the 
generalized Hubbard operator $\tilde{c}_{k \sigma}$ through a low-energy reduction procedure 12,15. In linear order in $1 / U$, the well known procedure of the canonical transformation [14,17] applied to the generalized Hubbard model, in the subspace of no double occupancy, leads to:

$$
\tilde{c}_{i \sigma}=c_{i \sigma}+\sum_{\delta} \frac{t_{\delta}}{U}\left(n_{i \bar{\sigma}} c_{i+\delta \sigma}-c_{i \bar{\sigma}}^{\dagger} c_{i} c_{i+\delta \bar{\sigma}}\right)
$$

Calling $|0\rangle\left(\left|\psi_{k}\right\rangle\right)$ the ground state of Eq. (幽) for the undoped (hole doped with wave vector $k$ ) system, and using the Lehmann representation of the wave function, one realizes that while the holon quasiparticle weight is:

$$
Z_{h}(k)=\left|\left\langle\psi_{k}\left|h_{k}^{\dagger}\right| 0\right\rangle\right|^{2}
$$

the weight for emitting a Hubbard electron is:

$$
Z_{c \sigma}^{G H}(k)=\left|\left\langle\psi_{k}\left|\tilde{c}_{k \sigma}\right| 0\right\rangle\right|^{2}+\left|\left\langle\psi_{k+Q}\left|\tilde{c}_{k \sigma}\right| 0\right\rangle\right|^{2}
$$

where $Q=(\pi, \pi)$, and $\left|\psi_{k}\right\rangle$ and $\left|\psi_{k+Q}\right\rangle$ are the degenerate eigenstates of lowest energy of Eq. (四) with a finite overlap with $\tilde{c}_{k \sigma}|0\rangle$. The corresponding result for the generalized $t-J$ model $Z_{c \sigma}^{G t J}(k)$ is obtained taking infinite $U$. Since $Z_{c \uparrow}(k)=Z_{c \downarrow}(k)$ we restrict to spin up in the following. The states $\left|\psi_{k}\right\rangle$ can be constructed following the procedure used by Reiter [6]. The only change in Eqs. 1 to 10 of Ref. [6], is that the quasiparticle energy $\lambda_{k}=$ $\lambda_{k+Q}$ is replaced by $\lambda_{k}-\epsilon_{k}$ in Eqs. 3, 6 and 9, and by $\lambda_{k}-\epsilon_{k-q}$ in Eq. 4 . Thus, writing explicitely only the terms with less than two spin-wave excitations we have:

$$
\left|\psi_{k}\right\rangle=A_{0}(k) h_{k}^{\dagger}|0\rangle+\frac{1}{\sqrt{N}} \sum_{q} A_{1}(k, q) h_{k-q}^{\dagger} \alpha_{q}^{\dagger}|0\rangle+\ldots
$$

where:

$$
A_{1}(k, q)=4 t_{1} M(k, q) G_{h}\left(k-q, \lambda_{k}-\omega_{q}\right) A_{0}(k) .
$$

Using Eqs. (2) and (6) and retaining only terms lines in spin deviations we obtain:

$$
\begin{aligned}
& \tilde{c}_{i \uparrow}=h_{i}^{\dagger}-\frac{t_{1}}{U}(1-x) \sum_{\eta} h_{i+\eta}^{\dagger} a_{i}^{\dagger}, \quad \text { if } i \in \mathrm{A} \\
& \tilde{c}_{i \uparrow}=h_{i}^{\dagger} a_{i}+\frac{1-x}{U}\left[t_{1} \sum_{\eta} h_{i+\eta}^{\dagger}+\sum_{\delta \neq \eta} t_{\delta} h_{i+\delta}^{\dagger}\left(a_{i+\delta}-a_{i}\right)\right], \quad i \in \mathrm{B} .
\end{aligned}
$$


The most important correction of order $1 / U$ is the first term between brackets in the second Eq. (11) and reflects the fact that in the ground state of the undoped Hubbard model, there is a finite double occupancy at sites B and an electron with spin up can be destroyed there, leaving a hole in one of its NN (this leads to the second term between brackets in Eqs. (12) and (14)).

Expressing Eqs. (11) in Fourier components, and using $\sum_{i \in \mathrm{A}(\mathrm{B})} e^{i k R_{i}}=\left(\delta_{k, 0}+\right.$ $\left.e^{i Q R_{i}} \delta_{k, Q}\right) N / 2$, we obtain:

$$
\tilde{c}_{k \uparrow}=\frac{1}{2}(1+f(k))\left(h_{k}^{\dagger}+s_{A} h_{k+Q}^{\dagger}\right)+\frac{1}{2 \sqrt{N}} \sum_{q}\left(h_{k+q}^{\dagger}-s_{A} h_{k+q+Q}^{\dagger}\right)\left[(1+g(k, q)) a_{q}-f(k) a_{q}^{\dagger}\right],
$$

where the phase $s_{A}=e^{i Q R_{i}}$ with $i \in \mathrm{A}$, and

$$
f(k)=\frac{t_{1}}{U}(1-x) \epsilon_{1}(k), \quad g(k, q)=\frac{1-x}{U}\left[t_{2}\left(\epsilon_{2}(k)-\epsilon_{2}(k+q)\right)+t_{3}\left(\epsilon_{1}(2 k)-\epsilon_{1}(2 k+2 q)\right)\right] .
$$

Using Eqs. (3), (7), (8), (9), (10) and (12) we obtain the desired result:

$$
\frac{Z_{c \sigma}^{G H}(k)}{Z_{h}(k)}=\frac{1}{2}\left|1+f(k)+\frac{8 t_{1}}{N} \sum_{q}^{\prime} M(k, q) G_{h}\left(k-q, \lambda_{k}-\omega_{q}\right)\left[v_{q}(1+g(k, q))-u_{q} f(k)\right]\right|^{2} .
$$

The sum is restricted to the magnetic Brillouin zone and the term with $q=0$ is excluded (there are no magnons with $q=0$ or $q=Q$ in the $\left|\psi_{k}\right\rangle$ ). The weight $Z_{c \sigma}^{G t J}$ for the generalized $t-J$ model operator $c_{i \sigma}$ is given by Eq. (14) with the Hubbard perturbative corrections $f(k)$ (first $\mathrm{NN}$ ) and $g(k, q)$ (second and third NN) set to zero.

In Fig. 1 we compare the weight for the $t-J$ model obtained by exact diagonalization $Z_{E D}^{t J}(k)$ in a square lattice of 20 sites [13] with our results $Z_{c \sigma}^{t J}(k)$ for the $20 \times 20$ cluster at equivalent wave vectors. The comparison between exact results for square clusters of 16, 18, 20 and 26 sites suggest that while the $Z_{E D}^{t J}(k)$ are nearly $20 \%$ larger for the $4 \times 4$ cluster, the finite size effects are of the order of $5 \%$ for larger clusters [13]. The agreement between the exact $Z_{E D}^{t J}(k)$ and SCBA $Z_{c \sigma}^{t J}(k)$ results is quite satisfactory. Note that the very small value 
of $Z^{t J}(Q)$ is a severe test to Eq. (14), since it requires a near cancellation of the different terms. Instead, the "bare" SCBA result satisfies $Z_{h}(k)=Z_{h}(k+Q)$ and cannot reproduce the shape of the exact results.

With the confidence gained by the above comparison, we have calculated the generalized $t-J$ and Hubbard weights for parameters which fit the observed quasiparticle dispersion $\lambda_{k}$ in $\mathrm{Sr}_{2} \mathrm{CuO}_{2} \mathrm{Cl}_{2}$ [7]. There are several choices of $t_{2}, t_{3}$ and $t^{\prime \prime}$, including different signs of $t^{\prime \prime}$ which produce nearly identical results. We took the parameters of Ref. [5]. The resulting dispersion and weights are represented in Fig. 2. Compared with the parameters of Fig. 1, the effects of $t_{2}, t_{3}$ and $t^{\prime \prime}$ are dramatic. They push the $\lambda_{k}$ towards the incoherent part of the spectrum and reduce considerably the weights for the lowest $\lambda_{k}$ (in the electron representation of Fig. 2). As a consequence, we could not detect quasiparticles near $k=0$, $Q$ or $(\pi, 0)\left(Z_{h}<10^{-4}\right.$ for these $\left.k\right)$. Therefore, the corresponding $\lambda_{k}$ are not represented in Fig. 2. The weights for the generalized $t-J$ and Hubbard models have significant differences: in contrast to the results for $t_{2}=t_{3}=t^{\prime \prime}=0$ (not shown), $Z_{c \sigma}^{G t J}(k)$ is larger for $k=(\pi / 2+\varepsilon, \pi / 2+\varepsilon)$ than for $k=(\pi / 2-\varepsilon, \pi / 2-\varepsilon)$ with small $\varepsilon$. Instead, $Z_{c \sigma}^{G H}(k)$, in agreement with experiment, is larger inside the non interacting Fermi surface. This effect is more noticeable for smaller values of $U\left(t_{1} / U=0.1\right.$ was taken in Fig. 2) [19].

In summary, using the SCBA and related wave function, we have calculated the dispersion and quasiparticle weight for removing a real electron in an undoped antiferromagnet described by a generalized $t-J$ or a generalized Hubbard model in the strong coupling limit. The weight for the $t-J$ model agrees very well with available exact results in sufficiently large clusters. While the generalized Hubbard can explain well both the measured dispersion and weight of the quasiparticle in $\mathrm{Sr}_{2} \mathrm{CuO}_{2} \mathrm{Cl}_{2}$, the generalized $t-J$ model, without mapping the electron operators, cannot.

One of us (FL) is supported by the Consejo Nacional de Investigaciones Científicas y Técnicas (CONICET), Argentina. (AAA) is partially supported by CONICET.

Note added: after submission of this manuscript we became aware of exact diagonalization results of the $t-J$ model in a square cluster of 32 sites with periodic boundary conditions 
which has 9 non-equivalent wave vectors [20]. (See Fig. 3). The dispersion relation $\lambda_{k}$ agrees very well with the SCBA results except at the points $k=(0,0),(\pi / 4, \pi / 4)$ and $(\pi, \pi / 2)$, where finite-size effects are obvious from the fact that $\lambda_{k} \neq \lambda_{k+Q}$. Except at $k=(0,0)$ and $(\pi / 4, \pi / 4)$, where the position of $\lambda_{k}$ affects the quasiparticle weights, these weigths are in excellent agreement with our results using Eq. (14). 


\section{REFERENCES}

[1] S. Schmitt-Rink, C.M. Varma, and A.E. Ruckenstein, Phys. Rev. Lett. 60, 2793 (1988).

[2] C.L. Kane, P.A. Lee, and N. Read, Phys. Rev. B 39, 6880 (1988).

[3] G. Martínez and P. Horsch, Phys. Rev. B 44, 317 (1991).

[4] Z. Liu and E. Manousakis, Phys. Rev. B 45, 2425 (1992).

[5] T. Xiang and J.M. Wheatley, Phys. Rev. B 54, R12653 (1996).

[6] G.F. Reiter, Phys. Rev. B 49, 1536 (1994).

[7] B.O. Wells, Z.-X. Shen, A. Matsuura, D.M. King, M.A. Kastner, M. Greven, and R.J. Birgeneau, Phys. Rev. Lett. 74, 964 (1995).

[8] A. Nazarenko, K.J.E. Vos, S. Haas, E. Dagotto, and R. Gooding, Phys. Rev. B 51, 8676 (1995).

[9] V.I. Belinicher, A.L. Chernyshev, and V.A. Shubin, Phys. Rev. B 54, 14914 (1996).

[10] F. Lema, J. Eroles, C.D. Batista and E. Gagliano, Phys. Rev. B (BUR574).

[11] O.A. Starykh, O.F. de Alcantara Bonfim, and G. Reiter, Phys. Rev. B 52, 12534 (1995).

[12] J. Eroles, C.D. Batista and A.A. Aligia, Physica C 261, 237 (1996); references therein. For large O-O hopping the resulting three-site term $t^{\prime \prime}$ favors a resonance-valence-bond superconducting state in the square lattice [C.D. Batista and A.A. Aligia, Physica C 261237 (1996); J. Low Temp. Phys. 105, 591 (1996)] and shifts towards lower values of $J$ the region of dominant superconducting correlations in one dimension [F. Lema, C.D. Batista and A.A. Aligia, Physica C 259, 287 (1996)].

[13] D. Poilblanc, T. Ziman, H.J. Schulz, and E. Dagotto, Phys. Rev. B 47, 14267 (1993).

[14] C.D. Batista and A.A. Aligia, Phys. Rev. B 47, 8929 (1993).

[15] L. Feiner, Phys. Rev. B 48, 16857 (1993). 
[16] J. Bala, A.M. Oleś, and J. Zaanen, Phys. Rev. B 52, 4597 (1995).

[17] H. Eskes, A.M. Oleś, M.B.J. Meinders, and W. Stephan, Phys. Rev. B 50, 17980 (1994); references therein.

[18] When comparing our results with those of Poilblanc et al., it should be taken into account that they took the opposite sign of $t_{1}$ (positive for holes), what is equivalent to a shift in $\mathbf{Q}=(\pi, \pi)$ of all wave vectors, and they summed over both spins, introducing a factor 2 with respect to our results. To compare with experiment the sign of $t_{1}$ should be determined by the mapping procedure from the three-band Hubbard model $H_{3 b}$ with original phases [12] (the usual change of phases of half of the orbitals of $H_{3 b}$ to have the same sign of the hoppings for all directions changes the sign of $\left.t_{1}\right)$. After this mapping, $c_{i \uparrow}^{\dagger}$ has the character of an effective electron creation operator (mainly of $\mathrm{O}$ character) over a vacuum state where all sites carry a Zhang-Rice singlet, and $t_{1} \sim 0.3-0.4 \mathrm{eV}$ $>0$ results.

[19] The effect of the correction term $t_{1} / U$ has been studied recently using finite size diagonalization by H. Eskes and R. Eder (preprint cond-mat / 9609233), with results which agree with ours.

[20] P.W. Leung and R.J. Gooding, Phys. Rev. B 52, R15711 (1995).

\section{FIGURE CAPTIONS}

Fig.1: Quasiparticle weight of the $t-J$ model $Z_{c \sigma}^{t J}(k)$ calculated with the SCBA in a $20 \times 20$ lattice for several wave vectors (triangles), compared with exact diagonalization results in

a square cluster of 20 sites $Z_{E D}^{t J}(k)$ [13,18] (squares), and the spinless holon weight $Z_{h}(k)$ of the SCBA (circles). Parameters are $t_{1}=1, J=0.3, t_{2}=t_{3}=t^{\prime \prime}=0$.

Fig.2: Top: quasiparticle dispersion in clusters of $16 \times 16$ (solid symbols) and $20 \times 20$ sites (open symbols). Bottom: corresponding generalized $t-J$ (squares) and generalized Hubbard (circles) quasiparticle weights. Parameters are: $t_{1}=0.35, t_{2}=-0.12, t_{3}=$ 
$0.08, J=0.15, t^{\prime \prime}=J / 4$ and $U=3.5$

Fig.3: Top: quasiparticle dispersion in the cluster of $16 \times 16$ (open squares) compared with the exact diagonalization results in a square cluster of 32 sites [20](solid triangles). Bottom: corresponding quasiparticle weights. 
Fig. 1 Lema

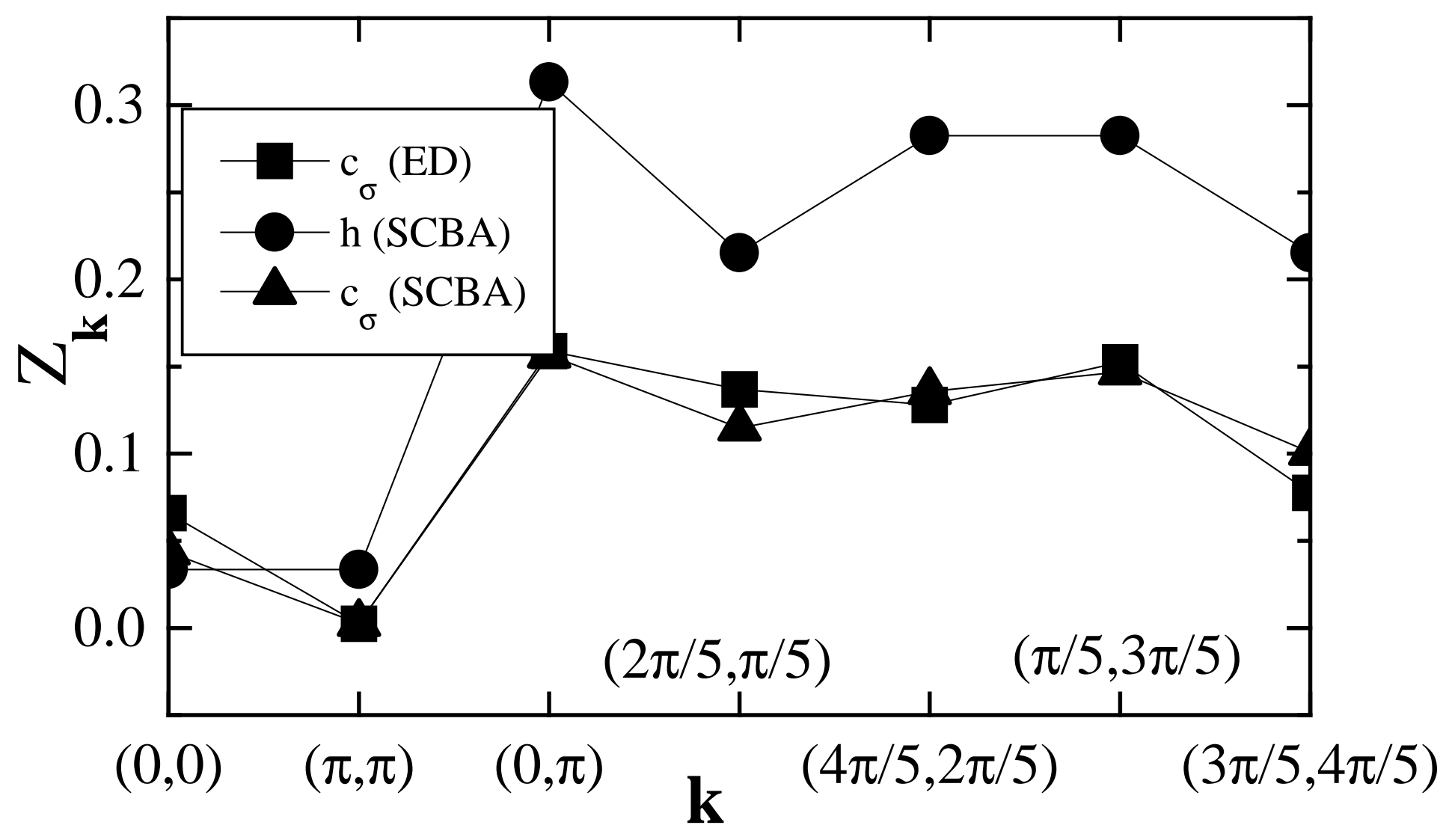




\section{Fig. 2 Lema}
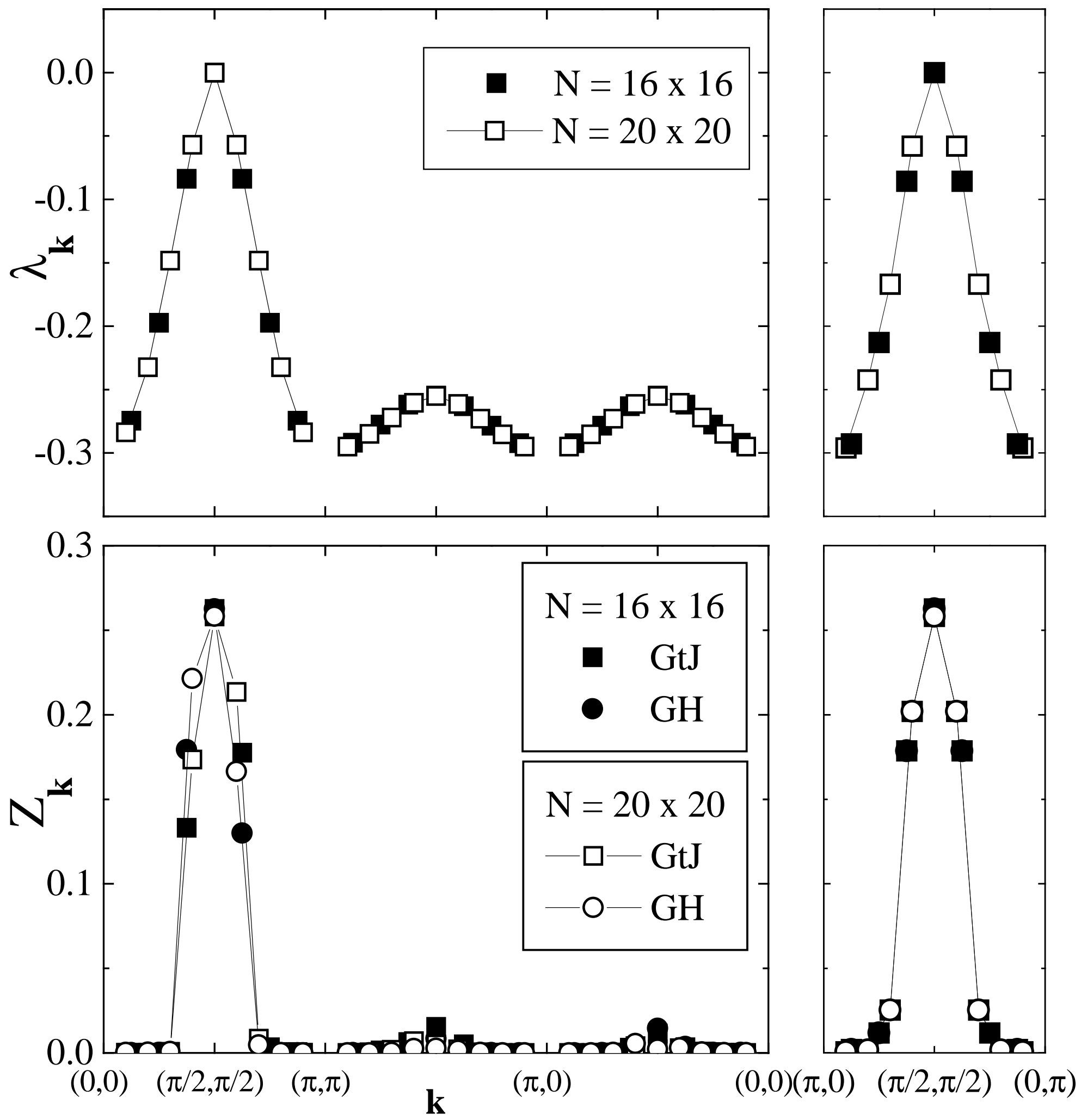
Fig. 3 Lema
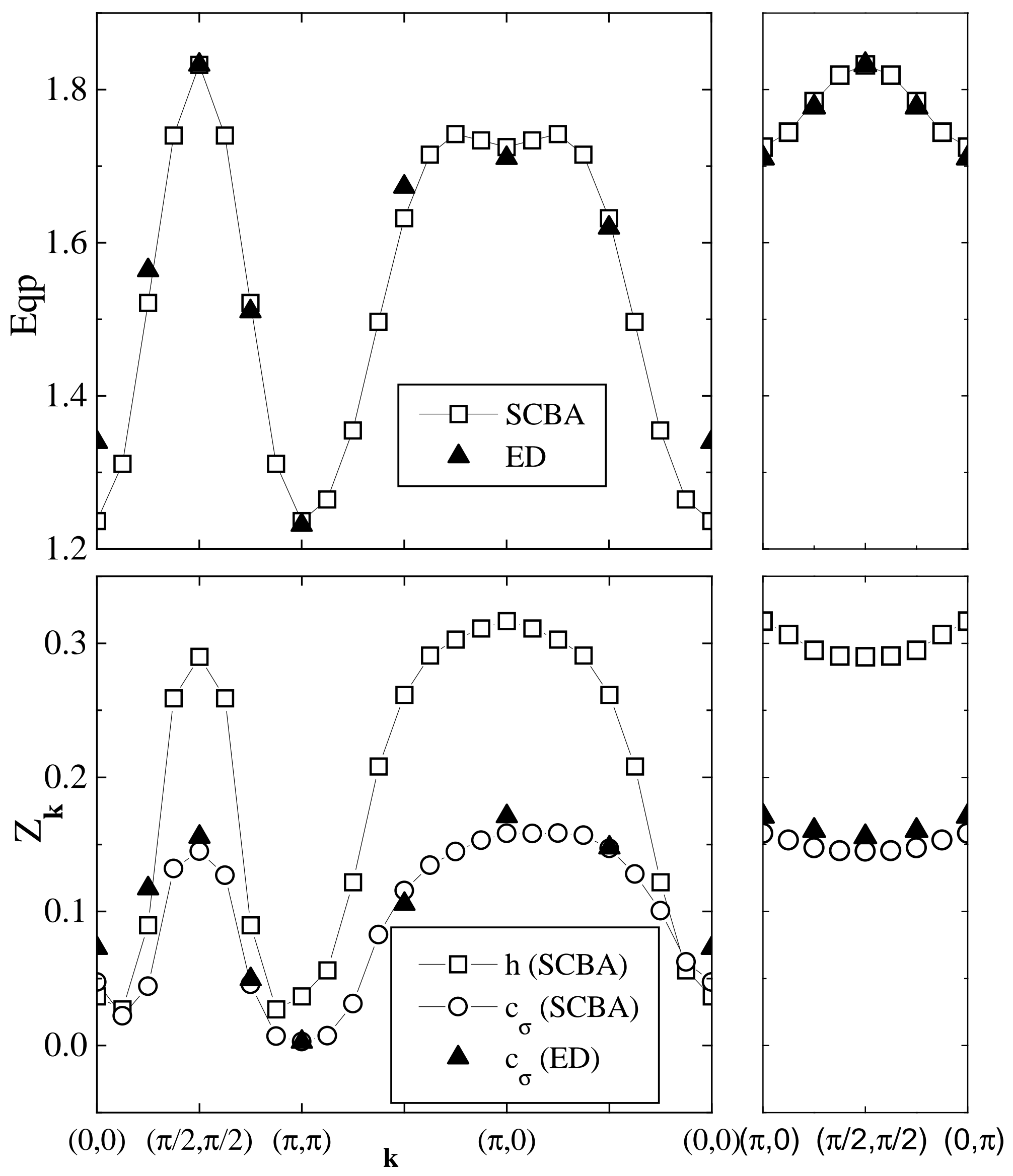